\title{
Pairing symmetry conversion by spin-active interfaces in superconducting junctions
}

\author{
Jacob Linder, ${ }^{1}$ Takehito Yokoyama, ${ }^{2}$ Asle Sudb $\varnothing,{ }^{1}$ and Matthias Eschrig ${ }^{3}$ \\ ${ }^{1}$ Department of Physics, Norwegian University of Science and Technology, N-7491 Trondheim, Norway \\ ${ }^{2}$ Department of Applied Physics, Nagoya University, Nagoya, 464-8603, Japan \\ ${ }^{3}$ Institut für Theoretische Festkörperphysik and DFG-Center for Functional Nanostructures, \\ Universität Karlsruhe, D-76128 Karlsruhe, Germany
}

(Dated: Received February 7, 2009)

\begin{abstract}
We study the proximity-induced superconducting correlations in a normal metal connected to a superconductor when the interface between them is spin-active and the normal metal is ballistic or diffusive. Remarkably, for any interface spin polarization there is a critical interface resistance, above which the conventional even-frequency proximity component vanishes completely at the chemical potential, while the odd-frequency component remains finite. We propose a way to unambiguously observe the odd-frequency component.

PACS numbers: 74.20.Rp, 74.50.+r, 74.20.-z
\end{abstract}

Superconductivity and superfluidity are hallmarks of the wave-like character of matter, and manifest themselves in vastly different systems, from ultracold dilute gases via cold metals and fluids, to extremely dense protonic and neutronic matter. In all these contexts, the symmetry of the order parameter is of profound importance. Over the last decades, the possibility of superconducting order parameters that change sign under a time-coordinate exchange of the two fermions comprising the Cooper-pair, has emerged in addition to the by now well studied varieties of orbital symmetries [1, 2, 3, 4, 5]. This so-called odd-frequency superconductivity [6] is distinct from the traditional even-frequency pairing in the Bardeen-CooperSchrieffer paradigm, and may be induced by proximity effects in hybrid structures of superconductors and magnets [1].

In a broader context, proximity systems offer the possibility of controlling the physics of competing broken symmetries. The fundamental heterostructure for studying proximity induced superconductivity is the superconductor/normal metal $(\mathrm{S} \mid \mathrm{N})$ bilayer, where the normal metal or the interface may have magnetic properties. Among possible triplet pair correlations, in the diffusive limit odd-frequency pairs are favored [7], whereas in ballistic hybrid systems both odd- and evenfrequency amplitudes compete [3, 4]. As all known superconductors to date exhibit an even-frequency order parameter, the observation of proximity induced effects that are particular to odd-frequency pairing would be of utmost interest.

There are two major difficulties associated with the detection of the odd-frequency state in superconductor/ferromagnet $(\mathrm{S} \mid \mathrm{F})$ bilayers. One is the usually short penetration depth into the ferromagnetic region, limited by the magnetic coherence length $\xi_{F}$, much less than the superconducting coherence length $\xi_{S}$ [1]. Another problem is that odd-frequency pairs are only well defined when even-frequency correlations vanish in the ferromagnet. Clear-cut signatures of the former are therefore only accessible in a limited parameter regime [8].

The majority of work on superconducting proximitystructures so far has been restricted to the diffusive limit and spin-inactive interfaces [9]. For a non-magnetic bilayer, a minigap appears in the density of states of the normal metal. It scales with the Thouless energy of the normal layer and with the transmission probability of the interface. Such minigap structures are readily accessible experimentally [10]. For a spin-active interface, the transmission properties of spin- $\uparrow$ and spin- $\downarrow$ electrons into a metal are different, and this gives rise to both spin-dependent conductivities and spin-dependent phase shifts at the interface [11, 12, 13, 14, 15]. In this Letter we show that a spin-active interface in a $\mathrm{S} \mid \mathrm{N}$ bilayer produces clear signatures of purely odd-frequency triplet pairing amplitudes that can be tested experimentally.

We consider the system shown in Fig. 1. The superconductor is conventional (even-frequency $s$-wave) while the interface is magnetic. We find that there is a dramatic change in the nature of proximity correlations when the spin-dependent phase shifts exceed the tunneling probability of the interface. The spin-active interface in an $\mathrm{S} \mid \mathrm{N}$ bilayer causes the evenfrequency correlations to vanish at zero excitation energy, while odd-frequency correlations appear. At the same time, the minigap, one of the hallmarks of the conventional proximity effect, is replaced by a low-energy band with enhanced density of states. We focus on the density of states (DOS) in the normal region, which can be probed by tunneling experiments. Our findings suggest that it should be possible to detect the odd-frequency amplitude without any interfering effects of even-frequency correlations. Since the exchange field is absent in the normal metal, this resolves the two main difficulties associated with the experimental detection of oddfrequency correlations mentioned above.

We adopt the quasiclassical theory of superconductivity [18], where information about the physical properties of the system is embedded in the Green's function. For equilibrium situations, it suffices to consider the retarded Green's func-

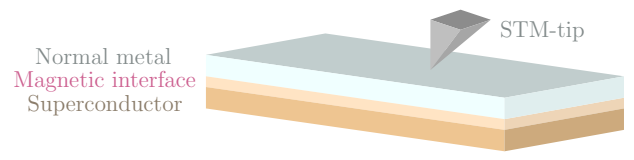

FIG. 1: (Color online) Proposed experimental setup for observation of the odd-frequency component in a diffusive normal metal layer|superconductor junction. 
tion, $\hat{g}$, that is parameterized conveniently in the normal $(\mathrm{N})$ region by a parameter $\theta_{\sigma}$, allowing for both singlet and triplet correlations [8]. In the superconducting (S) region, we employ the bulk solution $\hat{g}_{S}=c \cdot \underline{\tau_{3}} \otimes \underline{\sigma_{0}}+s \cdot \underline{\tau_{1}} \otimes\left(\underline{1} \sigma_{2}\right)$, with $c=\cosh (\theta), s=\sinh (\theta), \theta=\operatorname{atanh}(\Delta / \varepsilon), \overline{\tau_{i}}$ and $\overline{\sigma_{i}}$ being Pauli matrices in particle-hole and spin space, respectively.

We use the formalism described in Ref. [8], and consider first the diffusive limit. Then, the orbital symmetry for all proximity amplitudes is reduced to $s$-wave and hence the singlet component always has an even-frequency symmetry while the triplet component has an odd-frequency symmetry. The Green's functions are subject to boundary conditions, which in the tunneling limit assume the following form at the $\mathrm{S} \mid \mathrm{N}$ interface [13, 15]: $2 \gamma d \hat{g}_{N} \partial_{x} \hat{g}_{N}=$ $\left[\hat{g}_{S}, \hat{g}_{N}\right]+1\left(G_{\phi} / G_{T}\right)\left[\underline{\tau_{0}} \otimes \underline{\sigma_{3}}, \hat{g}_{N}\right]$, and at the outer interface $\operatorname{read} \partial_{x} \hat{g}_{N}=\hat{0}$. Here, $\gamma=R_{B} / R_{N}$ where $R_{B}\left(R_{N}\right)$ is the resistance of the barrier (normal region), and $d$ is the width of the normal region, while $G_{T}$ is the junction conductance in the normal-state. The boundary condition above contains an additional term $G_{\phi}$ compared to the usual non-magnetic boundary conditions in Ref. [9]. This term is due to spin-dependent phase shifts of quasiparticles being reflected at the interface. $G_{\phi}$ may be non-zero even if the transmission $G_{T} \rightarrow 0$, corresponding to a ferromagnetic insulator [13]. We define the superconducting coherence length $\xi_{S}=\sqrt{D / \Delta}$ and Thouless energy $\varepsilon_{\mathrm{Th}}=D / d^{2}$, where $D$ is the diffusion constant, and assume that the inelastic scattering length, $l_{\text {in }}$, is sufficiently large, such that $d \ll l_{\text {in }}$.

The Usadel equation [19] reads $D \partial_{x}^{2} \theta_{\sigma}+21 \varepsilon \sinh \theta_{\sigma}=0$, with boundary condition $\gamma d \partial_{x} \theta_{\sigma}=\left(c s_{\sigma}-\sigma s c_{\sigma}\right)+1 \sigma s_{\sigma} \frac{G_{\phi}}{G_{T}}$ at $x=0$ and $\partial_{x} \theta_{\sigma}=0$ at $x=d$. Here, $c_{\sigma}=\cosh \left(\theta_{\sigma}\right)$ and $s_{\sigma}=\sinh \left(\theta_{\sigma}\right)$. At zero energy, we find that the pairing amplitudes are either purely (odd-frequency) triplet,

$$
f_{s}(0)=0, f_{t}(0)=\frac{G_{T} \cdot \operatorname{sgn}\left(G_{\phi}\right)}{\sqrt{G_{\phi}^{2}-G_{T}^{2}}} \text { for } \frac{\left|G_{\phi}\right|}{G_{T}}>1,
$$

or purely (even-frequency) singlet

$$
f_{s}(0)=\frac{1 \cdot G_{T}}{\sqrt{G_{T}^{2}-G_{\phi}^{2}}}, f_{t}(0)=0 \text { for } \frac{\left|G_{\phi}\right|}{G_{T}}<1 .
$$

Thus, the presence of $G_{\phi}$ induces an odd-frequency component in the normal layer. The remarkable aspect of Eqs. (1) and (2) is that they are valid for any value of the width $d$ below the inelastic scattering length, and for any interface parameter $\gamma$. Thus, the vanishing of the singlet component is a robust feature in $\mathrm{S} \mid \mathrm{N}$ structures with spin-active interfaces, as long as $\left|G_{\phi}\right| / G_{T}>1$. Without loss of generality, we focus on positive values of $G_{\phi}$ from now on. The DOS is given as $N(\varepsilon) / N_{0}=\sum_{\sigma} \operatorname{Re}\left\{c_{\sigma}\right\} / 2$, yielding $N(0) / N_{0}=$ $\operatorname{Re}\left\{G_{\phi} / \sqrt{G_{\phi}^{2}-G_{T}^{2}}\right\}$. At zero-energy, the DOS thus vanishes as long as $G_{\phi} / G_{T}<1$, which means that the usual minigap in $\mathrm{S} \mid \mathrm{N}$ structures survives in this regime. However, the zero-energy DOS is enhanced for $G_{\phi} / G_{T}>1$ since the singlet component vanishes there.
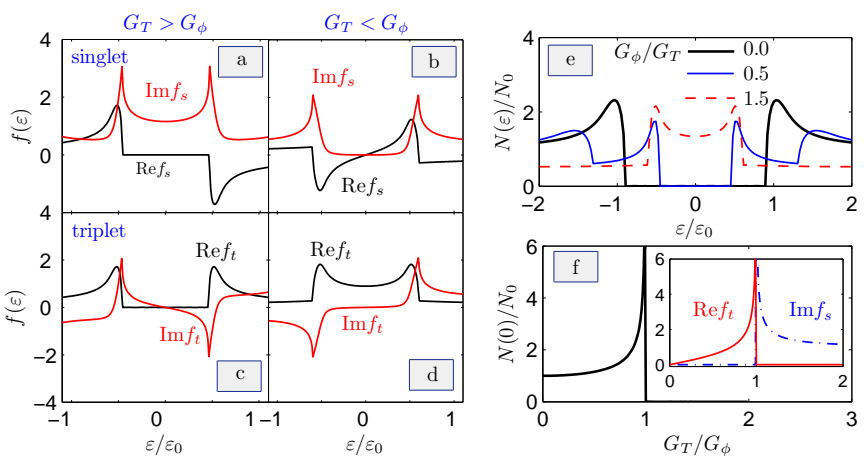

FIG. 2: (Color online) The singlet and triplet proximity amplitudes induced in the normal metal are shown for $G_{\phi} / G_{T}<1$ [in a) and c)] and $G_{\phi} / G_{T}>1$ [in b) and d)]. In e), we plot the energy-resolved DOS for several values of $G_{\phi} / G_{T}$. Finally, f) shows the zero-energy DOS as a function of $G_{T} / G_{\phi}$, with the proximity amplitudes shown in the inset.

The full energy-dependence of the DOS may only be obtained numerically. To model a realistic experimental setup, we fix $\gamma=10$ and $d / \xi_{S}=1.0$, although our qualitative results are independent of these particular choices. As a measure of the relevant energy scale, we define $\varepsilon_{0}=\varepsilon_{\mathrm{Th}} /(2 \gamma)$. The results are shown in Fig. 2 to investigate the effect of the spin-dependent phase shifts. The low-energy DOS is strongly enhanced due to the odd-frequency amplitude when $G_{\phi} / G_{T}>1\left(G_{\phi} / G_{T}=1.5\right.$ in the figure $)$. Conversely, the DOS develops a minigap around $\varepsilon=0$ when $G_{\phi} / G_{T}<1$ ( $G_{\phi} / G_{T}=0.5$ in the figure). The ratio $G_{\phi} / G_{T}$ depends on the microscopic barrier properties [15]. In the tunneling limit, one finds that $G_{\phi}$ can be considerably larger than $G_{T}$.

We suggest the following qualitative explanation for the mechanism behind the separation between even- and oddfrequency correlations. The superconductor induces a minigap $\propto G_{T}$ in the normal metal, while the spin-active barrier induces an effective exchange field $\propto G_{\phi}$. The situation in the normal metal then resembles that of a thin-film conventional superconductor in the presence of an in-plane external magnetic field [22], with the role of the gap and field played by $G_{T}$ and $G_{\phi}$, respectively. In that case, it is known that superconductivity is destroyed above the Clogston-Chandrasekhar limit [23], as the spin-singlet Cooper-pairs break up. In the present case, we observe coexistence of the exchange field and spin-singlet even-frequency superconductivity as long as $G_{\phi}$ is below the critical value of $G_{\phi}=G_{T}$. However, for $G_{\phi}>G_{T}$ spin-singlet pairing is no longer possible at the chemical potential. It is then replaced by spin-triplet pairing, which must be odd in frequency due to the isotropization of the gap in the diffusive limit. Thus, there is a natural separation between even-frequency and odd-frequency pairing in the normal metal at a critical value of the effective exchange field $G_{\phi}$.

The same effect occurs in the ballistic limit, as we now show. In this case, we can obtain the retarded Green's function using the formalism described in Refs. [14, 16]. The 
Eilenberger equation in the normal region reads $i v_{F x} \partial_{x} \hat{g}+$ $\left[\varepsilon \underline{\tau_{3}} \otimes \underline{\sigma_{0}}, \hat{g}\right]=\hat{0}$. For the boundary conditions, we use a scattering matrix describing the magnetic interface between the superconductor and the normal metal,

$$
\hat{S}=\left(\begin{array}{cc}
\underline{r}_{S} \cdot \exp \left(\frac{i}{2} \vartheta_{S}{\underline{\sigma_{3}}}\right) & \underline{t}_{S N} \cdot \exp \left(\frac{i}{2} \vartheta_{S N} \underline{\sigma}_{3}\right) \\
\underline{t}_{N S} \cdot \exp \left(\frac{i}{2} \vartheta_{N S} \underline{\sigma_{3}}\right) & -\underline{r}_{N} \cdot \exp \left(\frac{i}{2} \vartheta_{N} \underline{\sigma_{3}}\right)
\end{array}\right),
$$

with real reflection and transmission spin matrices $\underline{r}_{S}, \underline{r}_{N}$, $\underline{t}_{S N}$, and $\underline{t}_{N S}$. The spin mixing angles $\vartheta_{S}, \vartheta_{N}, \vartheta_{S N}$, and $\vartheta_{N S}$ describe spin dependent scattering phases [11]. Neglecting spin flip scattering, the transmission and reflection amplitudes are diagonal in spin space, and the relations $\underline{r}_{S}=\underline{r}_{N} \equiv$ $\operatorname{diag}\left[r_{\uparrow}, r_{\downarrow}\right], \underline{t}_{N S}=\underline{t}_{S N} \equiv \operatorname{diag}\left[t_{\uparrow}, t_{\downarrow}\right], r_{\uparrow}^{2}+t_{\uparrow}^{2}=r_{\downarrow}^{2}+t_{\downarrow}^{2}=1$, $\vartheta_{N S}+\vartheta_{S N}=\vartheta_{S}+\vartheta_{N}$ follow from the unitarity of $\hat{S}$. Possible scalar phases are omitted in Eq. (3), as they play no role in the final results.

We next concentrate on subgap energies. The anomalous amplitudes can be decomposed into singlet and triplet components, $f=\left(f_{s}+f_{t} \underline{\sigma_{3}}\right)\left(i \underline{\sigma_{2}}\right)$. Defining $f_{\sigma}=$ $\left(f_{s}+\sigma f_{t}\right) / 2$, we obtain on the top of the normal overlayer $(x=d) f_{\sigma}(\varepsilon)=-\operatorname{sgn}\left(\alpha_{\sigma}\right) t_{\uparrow} t_{\downarrow} / \sqrt{\alpha_{\sigma}^{2}-\left(t_{\uparrow} t_{\downarrow}\right)^{2}}$ with $\alpha_{\sigma}=\sin \left(2 \varepsilon d / v_{F x}+\sigma \vartheta_{+}\right)+r_{\uparrow} r_{\downarrow} \sin \left(2 \varepsilon d / v_{F x}+\sigma \vartheta_{-}\right)$. Here, $\vartheta_{ \pm}=\frac{1}{2}\left(\vartheta_{N} \pm \vartheta_{S}\right) \pm \arcsin (\varepsilon / \Delta)$, and $\varepsilon$ has to be supplemented by an infinitesimally small positive imaginary part. The interface parameters and the Fermi velocity component in $x$-direction, $v_{F x}=v_{F} \cos \psi$, depend on the impact angle $\psi$. The relevant energy scale in the problem is the ballistic Thouless energy, $\varepsilon_{T h}=v_{F} / 2 d$. For zero spin mixing angles we recover the known DOS for a normal state overlayer on a singlet superconductor. The DOS is non-zero only for $|\alpha|>t_{\uparrow} t_{\downarrow}$, which for sufficiently large impact angle always is fulfilled. Clearly, the most interesting regime concerns $\varepsilon / \varepsilon_{T h} \sim\left|\vartheta_{ \pm}\right| \sim t_{\uparrow} t_{\downarrow}$.

In the tunneling limit, for small excitation energies $\varepsilon / \varepsilon_{T h} \ll 1$ and small spin mixing angles $\vartheta_{ \pm}$we obtain $\alpha_{\sigma}=\left(4 \varepsilon d / v_{F x}+\sigma \vartheta_{N}\right)$. In this case, due to $\vartheta_{+}+\vartheta_{-}=\vartheta_{N}$, only the spin mixing angle for reflection at the normal side of the interface enters, and acts as an effective exchange field $b=\vartheta_{N} v_{F x} / 4 d$ on the quasiparticles. Especially interesting is the case $\varepsilon=0$, for which all proximity amplitudes are even in momentum. For $\varepsilon=0$ we obtain $\alpha_{\sigma}=\sigma \vartheta_{N}$, and the pairing amplitudes are either purely (odd-frequency) triplet,

$$
f_{s}(0)=0, f_{t}(0)=\frac{-t_{\uparrow} t_{\downarrow} \cdot \operatorname{sgn}\left(\vartheta_{N}\right)}{\sqrt{\vartheta_{N}^{2}-\left(t_{\uparrow} t_{\downarrow}\right)^{2}}} \text { for } \frac{\left|\vartheta_{N}\right|}{t_{\uparrow} t_{\downarrow}}>1,
$$

or purely (even-frequency) singlet

$$
f_{s}(0)=\frac{1 \cdot t_{\uparrow} t_{\downarrow}}{\sqrt{\left(t_{\uparrow} t_{\downarrow}\right)^{2}-\vartheta_{N}^{2}}}, f_{t}(0)=0 \text { for } \frac{\left|\vartheta_{N}\right|}{t_{\uparrow} t_{\downarrow}}<1 .
$$

Comparing with the results for the diffusive case, we find that $G_{\phi} / G_{T}$ corresponds to $-\vartheta_{N} /\left(t_{\uparrow} t_{\downarrow}\right)$.

In Fig. 3, we show results for the proximity amplitudes in the ballistic limit, and focus on positive values of $\vartheta_{N}$ without loss of generality. A systematic expansion of all terms in the

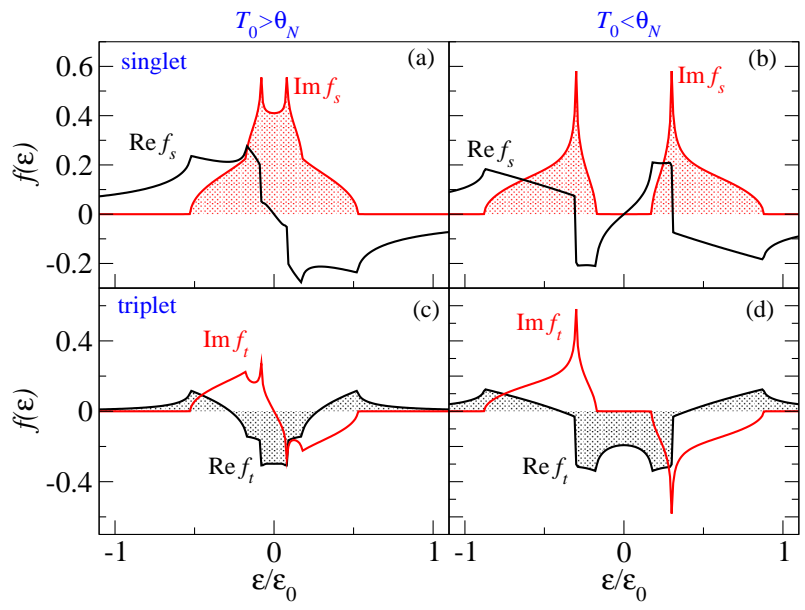

FIG. 3: (Color online) Momentum-averaged proximity amplitudes at the surface of the normal layer. Parameters: $d=v_{F} / \Delta, T_{0}=0.1$ (see text). (a) and (c): $\vartheta_{N}=\vartheta_{S}=0.05<T_{0}$; (b) and (d): $\vartheta_{N}=\vartheta_{S}=0.15>T_{0}$. Energy units are $\varepsilon_{0}=T_{0} \varepsilon_{T h}$. Even frequency singlet components are shown in (a-b), odd frequency triplet components in (c-d).
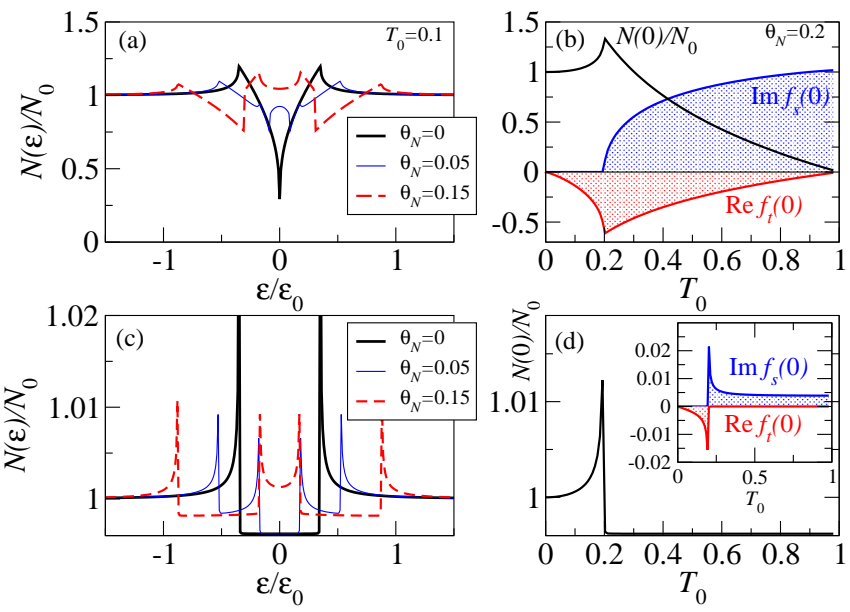

FIG. 4: (Color online) (a) DOS as function of energy at the top of the normal layer for fixed transmission probability $T_{0}=0.1$, and various values of $\vartheta_{N}=\vartheta_{S}$. Remaining parameters are as in Fig. 3 (b) DOS and proximity amplitudes at $\varepsilon=0$ for $\vartheta_{N}=\vartheta_{S}=0.2$ as function of $T_{0}$. In (c) and (d) we show the results corresponding to (a) and (b) when assuming an (abrupt) tunneling cone with opening angle of 10 degree.

tunneling probability shows that in the tunneling limit the spin dependence of the transmission probabilities can be neglected, and only that of the phase shifts needs to be kept. Thus, we assume $t_{\uparrow}=t_{\downarrow}=t$. We model the dependence on the impact angle $\psi$ as $t(\mu)=\left(t_{0}\right)^{\frac{1}{\mu}}, \mu=\cos \psi$, and assume for simplicity spin mixing angles independent of $\mu$. The tunneling probability for normal impact is $T_{0}=t_{0}^{2}$. In the case $T_{0}<\vartheta_{N}$ at small energies the odd frequency triplet amplitude dominates, and it is the only non-zero amplitude at $\varepsilon=0$. On the other hand, for $T_{0}>\vartheta_{N}$ both singlet and triplet amplitudes contribute. This is due to the fact that for large impact angles the 
transmission probability $t(\mu)^{2}$ drops below the value for the spin mixing angle $\vartheta_{N}$.

We turn now to the DOS. The general expression, assuming the bulk solution in the superconductor, is $N(\varepsilon) / N_{0}=$ $\operatorname{Re} \sum_{\sigma= \pm 1} \int_{0}^{1}\left|\alpha_{\sigma}\right| / \sqrt{\alpha_{\sigma}^{2}-\left(t_{\uparrow} t_{\downarrow}\right)^{2}} \quad d \mu$. In the tunneling limit, this simplifies again, and provided that $\left|\vartheta_{N}\right|>$ $t_{\uparrow} t_{\downarrow}$ for all impact angles, the DOS at the Fermi level is enhanced above its normal state value, $N(0) / N_{0}=$ $\int d \mu\left|\vartheta_{N}\right| / \sqrt{\vartheta_{N}^{2}-\left(t_{\uparrow} t_{\downarrow}\right)^{2}}$. In Fig. 4, we show results for the DOS. In (a-b) we assume the dependence on the impact angle as above, whereas in (c-d) we allow tunnelling only in a narrow tunneling cone of 10 degrees. The DOS for the cases of dominating triplet amplitudes and dominating singlet amplitudes differ qualitatively. In the case of a tunneling cone this difference is most drastic, and a comparison with the results above shows that it is very similar to the diffusive case. In the right panels, where $\vartheta_{N}=\vartheta_{S}=0.2$, we demonstrate that for $T_{0}<0.2$ only the odd frequency triplet amplitude is present at the chemical potential, while the singlet amplitude is zero. The corresponding zero-bias DOS is enhanced in this region, whereas it is reduced in the region when singlet correlations are present at $\varepsilon=0$.

The simplest experimental manifestation of the oddfrequency component is a zero-energy peak in the DOS [17, 20, 21]. In $S \mid F$ layers, where this phenomenon has been discussed previously, a clear zero-energy peak is unfortunately often masked by the simultaneous presence of singlet correlations $f_{s}$, which tend to suppress the DOS at low energies. This is not so in the system we consider, provided only $T_{0}<\left|\vartheta_{N}\right|$ in the ballistic limit, or equivalently, $G_{T}<\left|G_{\phi}\right|$ in the diffusive limit. This is ideal for a direct observation of the odd-frequency component, manifested as a zero-energy peak in the DOS.

The important factor, with regard to isolation of the oddfrequency correlations at zero energy is the interface. The even-frequency correlations vanish completely when the interface transmission is sufficiently low. The parameters $\vartheta_{N}$, or equivalently, $G_{\phi}$ can be increased by increasing the magnetic polarization of the barrier separating the superconducting and normal layers. By fabricating several samples with progressively increasing strength of magnetic moment of the barrier, one should be able to observe an abrupt crossover at the zero-energy DOS above a certain strength of the magnetic moment. Alternatively, one could alter the interface transmission by varying the thickness of the insulating region.

In summary, we have investigated the proximity-effect in a superconductor/normal metal bilayer with spin-active interface. We find that both in the ballistic and diffusive limits, the usual even-frequency correlations may vanish completely at zero energy, while odd-frequency correlations persist. This result is completely independent of the specific values for the layer thicknesses and barrier resistances, indicating that it is a robust and general feature of spin-active interfaces. Our findings suggest a way of obtaining unambiguous experimental identification of superconducting odd-frequency correlations.

Acknowledgments. A. Cottet and D. Huertas-Hernando are thanked for helpful communications. J.L. and A.S. were supported by the Norwegian Research Council Grants No. 158518/431 and No. 158547/431 (NANOMAT), and Grant No. 167498/V30 (STORFORSK). T.Y. was supported by the JSPS.

[1] F. S. Bergeret et al., Rev. Mod. Phys. 77, 1321 (2005).

[2] A. I. Buzdin, Rev. Mod. Phys. 77, 935 (2005).

[3] Y. Tanaka et al., Phys. Rev. Lett. 99, 037005 (2007); Y. Tanaka and A. A. Golubov, Phys. Rev. Lett. 98, 037003 (2007).

[4] M. Eschrig et al., J. Low Temp. Phys. 147457 (2007).

[5] T. Yokoyama et al., Phys. Rev. B 78, 012508 (2008).

[6] V. L. Berezinskii, JETP Lett. 20, 287 (1974).

[7] A. Volkov et al., Phys. Rev. Lett. 90, 117006 (2003)

[8] J. Linder et al., Phys. Rev. B 77, 174514 (2008).

[9] M. Yu. Kupriyanov and V. F. Lukichev, Zh. Exp. Teor. Fiz. 94, 139 (1988) ; Yu. Nazarov, Superlatt.Microstruct. 25, 1221 (1999).

[10] H. le Sueur et al., Phys. Rev. Lett. 100, 197002 (2008).

[11] T. Tokuyasu et al., Phys. Rev. B 38, 4504 (1988); A. Millis et al., Phys. Rev. B 38, 4504 (1988).

[12] A. Brataas et al., Phys. Rev. Lett. 11, 2481 (2000); A. Brataas et al., Phys. Rep. 427, 157 (2006).

[13] D.H. Hernando et al., Phys. Rev. Lett. 88, 047003 (2002).

[14] M. Eschrig et al., Phys. Rev. Lett. 90, 137003 (2003); M. Eschrig and T. Löfwander, Nature Physics 4, 138 (2008).

[15] A. Cottet and W. Belzig, Phys. Rev. B 72, 180503 (2005).

[16] M. Eschrig, Phys. Rev. B 61, 9061 (2000); M. Fogelström, Phys. Rev. B 62, 11812 (2000); E. Zhao et al., Phys. Rev. B 38, 134510 (2004).

[17] Y. Asano et al., Phys. Rev. Lett. 98, 107002 (2007).

[18] J. W. Serene and D. Rainer, Phys. Rep. 101, 221 (1983)

[19] K. Usadel, Phys. Rev. Lett. 25, 507 (1970).

[20] T. Yokoyama et al., Phys. Rev. B 75, 134510 (2007).

[21] V. Braude and Yu. V. Nazarov, Phys. Rev. Lett. 98, 077003 (2007).

[22] R. Meservey and P. M. Tedrow, Phys. Rep. 238, 173 (1994).

[23] A. M. Clogston, Phys. Rev. Lett. 9, 266 (1962); B. S. Chandrasekhar, Appl. Phys. Lett. 1, 7 (1962). 